\title{
New Tools for Measuring and Improving Patient Safety in Canadian Hospitals
}

Jennifer D’Silva, Joseph Emmanuel Amuah, Vanessa Sovran, Anne MacLaurin, Jennifer Rodgers, Tracy Johnson, Kira Leeb and Sandi Kossey

\begin{abstract}
The Canadian Institute for Health Information (CIHI) and the Canadian Patient Safety Institute (CPSI) have collaborated on a new measure of patient safety, along with a resource of evidence-informed practices. This measure captures four broad categories of harm in acute care hospitals, consisting of $\mathbf{3 1}$ clinical groups selected by clinicians. Analysis showed that harm was experienced in 1 of 18 hospital stays in Canada in 2014-2015 and that no single category accounted for the majority of harmful events. Although $\mathrm{CIHI}$ and CPSI continue to work with hospitals and experts to further refine the methodology, the measure and associated Improvement Resource are useful new tools for monitoring and identifying harm, and have the potential to improve patient safety.
\end{abstract}

\section{Introduction}

Since 2011, the Canadian Institute for Health Information (CIHI) and the Canadian Patient Safety Institute (CPSI) have been collaborating to develop a broad new measure of patient safety that is linked to evidence-informed practices for improvement. Although event-specific indicators and local data collection efforts do exist (CPSI 2015), there has been, to date, an information gap in answering high-level and broad questions about patient safety in Canadian hospitals. This new measure seeks to fill that gap and, by complementing existing patient safety initiatives, provide a more complete picture of harm occurring to patients in hospitals. The report Measuring Patient Harm in Canadian Hospitals (https://secure.cihi.ca/estore/productFamily. htm?locale $=$ en $\&$ pf $=$ PFC3312; CIHI and CPSI 2016) along with the Hospital Harm Improvement Resource (http://www.patientsafetyinstitute.ca/en/toolsResources/Hospital-Harm-Measure/ Improvement-Resources/Pages/default.aspx), were released by
CIHI and CPSI in October 2016 to introduce this new measure and provide a high-level overview of patient safety in Canadian hospitals.

\section{Methodology}

The new hospital harm measure captures acute care hospitalizations with at least one occurrence of unintended harm that could potentially have been prevented by implementing known evidence-informed practices. The harm results from healthcare received, rather than from the patient's underlying conditions. To be captured in the measure, the harm must have occurred after admission and required treatment or prolonged the hospital stay, and must fall within one of the 31 types of harm that make up the measure. Because this measure uses data which CIHI already regularly collects from hospitals, it will be relatively easy to update and will not require additional data collection.

The measure captures 31 different types of harm (or clinical groups) in four broad categories: healthcare-/ medication-associated conditions, healthcare-associated infections, patient accidents and procedure-associated conditions (Figure 1). These clinical groups were selected by clinicians because evidence shows that these types of harm can potentially be reduced by implementing evidence-informed practices. Although the measure captures a broad range of harm, it does not capture the following: near misses (incidents that did not reach the patient), incidents or events that reached the patient but did not cause harm, harm that was undetected during the hospital stay but surfaced after discharge and harm to patients outside of acute inpatient care (e.g., harm in the emergency department). 
Figure 1.

Hospital Harm Framework

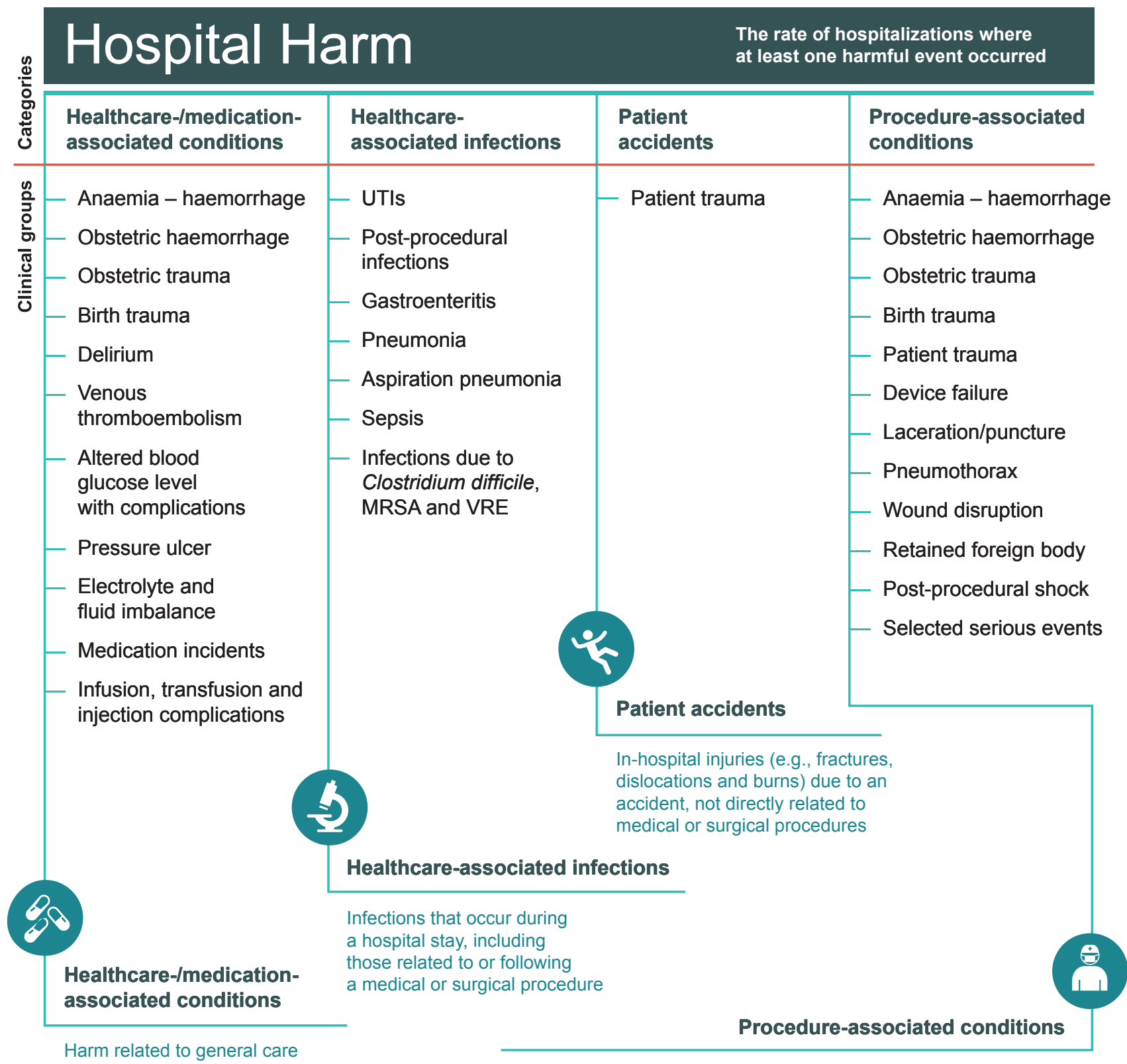

provided and/or medication

administered during a hospital stay
- Misadventures to patients during surgical and medical procedures

- Events associated with medical devices used for diagnosis and treatment

- Abnormal reactions or complications of surgical or medical procedures

\section{Category}

The number of hospitalizations with at least one harmful event in that category

\section{Clinical group}

The number of hospitalizations with at least one harmful event in that clinical group

MRSA = methicillin-resistant Staphylococcus aureus; UTI = urinary tract infection; VRE = vancomycin-resistant enterococci.

In the Hospital Harm Framework, each occurrence of harm is captured only once for each patient, even though the event may fall into more than one clinical group. This is to prevent overestimating harm if a patient's condition were included in more than one clinical group. Thus, a UTI after surgery is counted in the UTI clinical group, not in the post-procedural infection group. The exception to this rule is infections due to MRSA or VRE, as these could also be captured in other clinical groups. However, if a patient experienced more than one type of harmful event (e.g., a UTI and a fall), each event would be counted within its respective clinical group, but only once in the overall rate of hospital harm. See Measuring Patient Harm in Canadian Hospitals: Technical Report for details (CIHI 2016). 


\section{Key Findings}

Harm was experienced in one of every 18 hospital stays in Canada in 2014-2015. Overall, 138,000 or 5.6\% of all hospitalizations during this time involved at least one occurrence of harm, with the rate staying constant over the past three years. One in five of these hospitalizations involved more than one occurrence of harm. There were no patterns found for patients experiencing more than one occurrence.

No single category of harm accounted for a majority of events. Healthcare-associated infections and healthcare-/ medication-associated conditions each accounted for $37 \%$ of harm, procedure-associated conditions accounted for $23 \%$ and patient accidents $3 \%$. The five most common types of harm, making up 51\% of all harmful events, were: electrolyte and fluid imbalance, urinary tract infections, delirium, anaemia-haemorrhage and pneumonia (Figure 2).

Figure 2.

Distribution of harmful events by clinical group

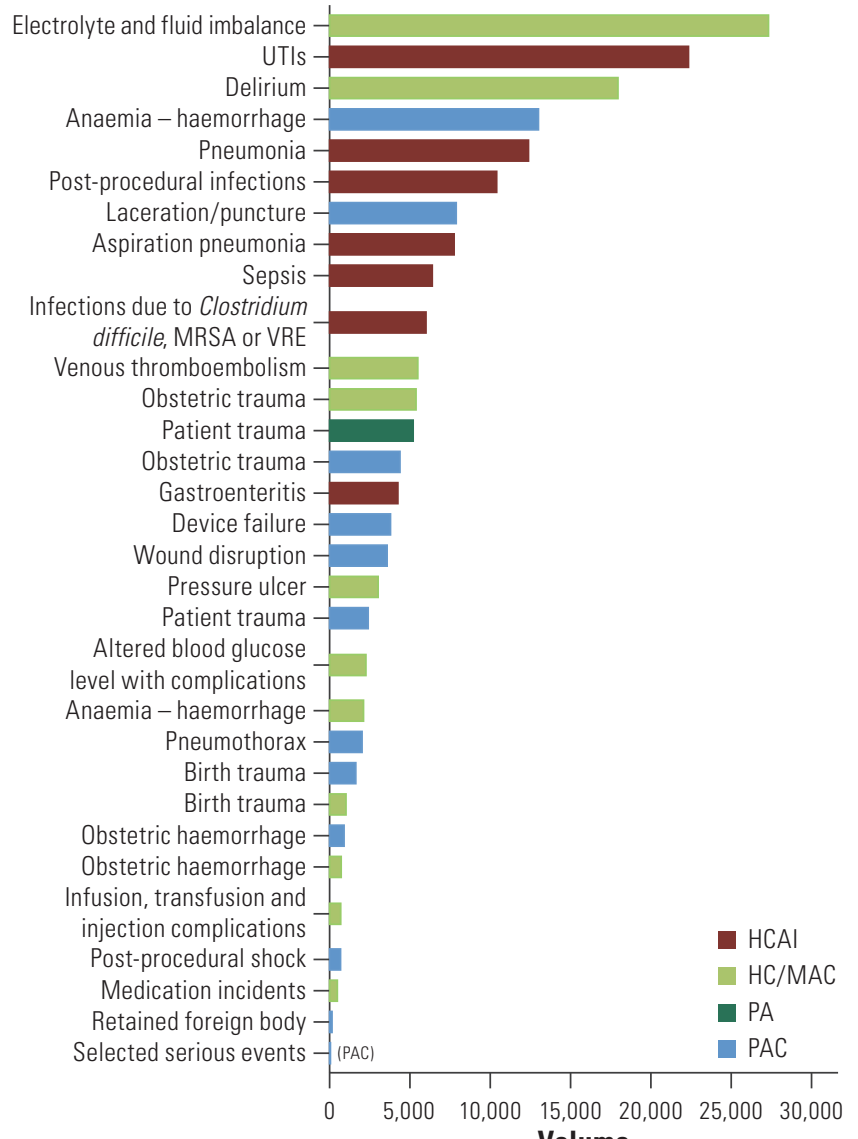

Volume

$\mathrm{HC} / \mathrm{MAC}=$ healthcare-/medication-associated conditions; $\mathrm{HCAl}=$ healthcare-associated infections; MRSA = methicillin-resistant Staphylococcus aureus; $\mathrm{PA}=$ patient accidents; $\mathrm{PAC}=$ procedureassociated conditions; UTI = urinary tract infection; VRE = vancomycin-resistant enterococci.

Data from Quebec as well as data for some mental health patients have been excluded owing to methodological issues.

Source: Discharge Abstract Database, 2014-2015, CIHI.
Patients who were more complex were more likely to experience a harmful event, and were also more likely to experience more than one harmful event. Complex patients were people with diseases in addition to the one for which they were being hospitalized, and/or those who were treated by more than one type of physician during their hospital stay.

It was estimated that patients who experienced harm spent over half a million additional days in hospital: that is more than 1,600 beds each day, or the equivalent of approximately four large hospitals occupied over a year. The associated hospital cost (excluding physician fees, follow-up care or readmission) is estimated to be $\$ 685$ million in $2014-2015$. Although it may not be possible to recover all these resources, even a small percentage saved could be used to address other unmet healthcare needs.

The Hospital Harm Improvement Resource, developed by CPSI, is a compilation of evidence-informed practices and resources for each of the 31 clinical groups included in the hospital harm measure. By making an array of patient safety information easily available, hospitals are aided in optimizing patient care. This online tool, which links measurement and improvement to support hospitals in their improvement efforts, will be updated regularly as new evidence emerges.

\section{Discussion and Conclusion}

The hospital harm measure and Improvement Resource are useful new tools for monitoring rates of harm and identifying potential issues for further investigation. Used in conjunction with other patient safety information available to hospitals such as patient safety incident reports, patient experience surveys, infection control data and global trigger tools, these new tools can provide a richer and more complete picture of patient safety in Canadian hospitals and help make hospitals safer.

Although healthcare providers strive to deliver the safest care possible, it may not be possible to prevent every occurrence of harm, and so hospital harm rates may never reach zero. Aspiration pneumonia provides an example: this may occur in patients who have difficulty swallowing - food may pass inadvertently into the lungs and cause pneumonia (Armstrong and Mosher 2011). Although the risk can be reduced by offering smaller bites or avoiding medications that dry out the mouth (Metheny 2012), it may be unavoidable in some instances. Even if harmful events may never reach zero, the types of harm included in the measure can potentially be reduced by following evidence-informed practices.

$\mathrm{CIHI}$ and CPSI continue to work with hospitals, clinical leaders and patient safety experts to refine the methodology for capturing hospital harm and to modify it to provide greater accuracy, including investigating risk adjustment. To this end, updated technical notes (http://indicatorlibrary.cihi.ca/ download/attachments/10453027/Hospital Harm Appendices. pdf? version $=1 \&$ modificationDate $=1486395477000 \&$ api $=\mathrm{v} 2$ ) 
were released in February 2017 (CIHI 2017). The feasibility of developing this measure into a facility-level indicator, enabling hospital comparisons, will continue to be assessed. Related indicators on obstetric trauma and sepsis are already available on CIHI's website Your Health System - In Depth (https://yourhealthsystem.cihi.ca/hsp/indepth?lang=en).

It is important to remember the impact of hospital harm on patients and their families. After the death of her father because of multiple complications following surgery, Carole Jukosky, of Patients for Patient Safety Canada, is working hard to share a message with all care providers (CPSI 2014):

\footnotetext{
"We went into healthcare for a reason. Don't forget what that reason is and always think with your hearts and use compassion."
}

Safer healthcare is possible for all Canadians, and the new hospital harm measure and Improvement Resource are tools that can aid health system leaders and care providers alongside their current quality improvement efforts. Armed with evidenceinformed practices, informed by continued research efforts and united through collaboration, clinicians, hospital staff and patients all have a role to play in improving safety in Canadian hospitals.

Additional information on the hospital harm measure and the Improvement Resource can be found on CIHI and CPSI's websites. HQ

\section{Acknowledgements}

The authors wish to acknowledge contributions to the CIHI and CPSI report Measuring Patient Harm in Canadian Hospitals, on which this article was based. In particular, the Emerging Issues and Health System Performance teams at CIHI are acknowledged, especially Chantal Couris, Farhat Farrokhi, Jennifer Frood, Yanyan Gong, Jenny Lineker, Ben Reason and Saad Shakeel.

\section{References}

Armstrong, J.R. and B.D. Mosher. 2011. "Aspiration Pneumonia after Stroke: Intervention and Prevention." The Neurohospitalist 1(2): 85-93. doi:10.1177/1941875210395775.

Canadian Institute for Health Information (CIHI). 2016. Measuring Patient Harm in Canadian Hospitals: Technical Report. Ottawa, ON: Author. Retrieved February 24, 2017. <https://www.cihi.ca/sites/ default/files/document/hospital_harm_technical_notes_en.pdf>.
Canadian Institute for Health Information (CIHI). 2017. Hospital Harm Indicator: Appendices to Indicator Library. Ottawa, ON: Author. Retrieved February 24, 2017. <http://indicatorlibrary.cihi.ca/download/ attachments/10453027/Hospital\%20Harm\%20Appendices.pdf?versio $\mathrm{n}=1 \&$ modificationDate $=1486395477000 \& a \mathrm{a} i=\mathrm{v} 2>$.

Canadian Institute for Health Information and Canadian Patient Safety Institute (CIHI and CPSI). 2016. Measuring Patient Harm in Canadian Hospitals. Ottawa, ON: Author. Retrieved February 24, 2017. <https://www.cihi.ca/sites/default/files/document/cihi_cpsi_ hospital_harm_en.pdf>.

Canadian Patient Safety Institute (CPSI). 2014. Father's Death Fuels Quest for Healthcare Improvement. Retrieved March 31, 2017. [Patient Stories Video Series]. <http://www.patientsafetyinstitute.ca/en/ toolsResources/Member-Videos-and-Stories/Pages/Carole-Jukosky. aspx>.

Canadian Patient Safety Institute (CPSI). 2015. Patient Safety and Quality Priorities for Consortium Participants. Retrieved April 5, 2017. <http://www.patientsafetyinstitute.ca/en/toolsResources/PatientSafety-Quality-Priorities-Snap-Shot/Documents/Canadian\%20Scan_ Final_English.pdf>.

Metheny, N.A. 2012. Preventing Aspiration in Older Adults with Dysphagia. Retrieved February 24, 2017. <https://consultgeri.org/ try-this/general-assessment/issue-20.pdf>.

\section{About the Authors}

Jennifer D'Silva is manager of Emerging Issues at the Canadian Institute for Health Information, Toronto, ON.

Joseph Emmanuel Amuah is senior researcher of Health System Performance at the Canadian Institute for Health Information, Ottawa, ON.

Vanessa Sovran is coordinator of Emerging Issues at the Canadian Institute for Health Information, Toronto, ON.

Anne MacLaurin is patient safety improvement lead at the Canadian Patient Safety Institute, Charlottetown, PE.

Jennifer Rodgers was patient safety improvement lead at the Canadian Patient Safety Institute, Barrie, ON

Tracy Johnson is director of Health System Analysis and Emerging Issues at the Canadian Institute for Health Information, Toronto, ON.

Kira Leeb is director of Health System Performance at the Canadian Institute for Health Information, Toronto, ON.

Sandi Kossey is senior director of Strategic Partnerships and Priorities at the Canadian Patient Safety Institute, Edmonton, AB.

\section{electronichealthcare.net}

